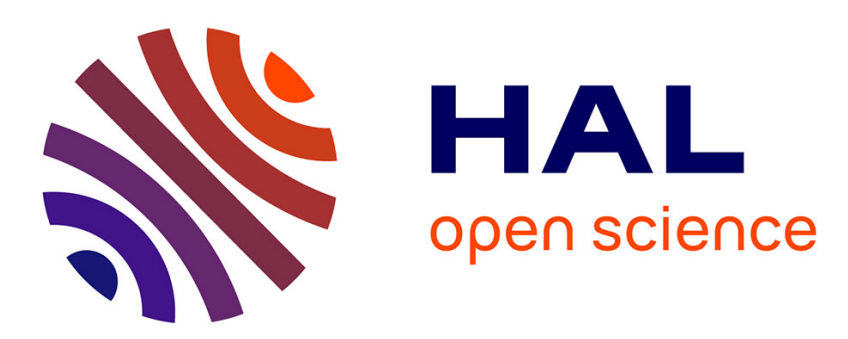

\title{
Interacting damage models mapped onto Ising and percolation models
}

\author{
Renaud Toussaint, Steven R Pride
}

\section{To cite this version:}

Renaud Toussaint, Steven R Pride. Interacting damage models mapped onto Ising and percolation models. Physical Review E: Statistical, Nonlinear, and Soft Matter Physics, 2005, 71 (4), pp.046127. 10.1103/physreve.71.046127 . hal-00107357

\section{HAL Id: hal-00107357 https://hal.science/hal-00107357}

Submitted on 20 Nov 2018

HAL is a multi-disciplinary open access archive for the deposit and dissemination of scientific research documents, whether they are published or not. The documents may come from teaching and research institutions in France or abroad, or from public or private research centers.
L'archive ouverte pluridisciplinaire HAL, est destinée au dépôt et à la diffusion de documents scientifiques de niveau recherche, publiés ou non, émanant des établissements d'enseignement et de recherche français ou étrangers, des laboratoires publics ou privés. 


\title{
Interacting damage models mapped onto Ising and percolation models
}

\author{
Renaud Toussaint* \\ Department of Physics, University of Oslo, P. O. Box 1043 Blindern, 0316 Oslo, Norway \\ Steven R. Pride \\ Earth Science Division, Lawrence Berkeley National Laboratory, 1 Cyclotron Road, MS 90-1116, Berkeley, California 94720, USA
}

(Received 16 March 2004; revised manuscript received 1 February 2005; published 21 April 2005)

\begin{abstract}
We introduce a class of damage models on regular lattices with isotropic interactions between the broken cells of the lattice. Quasistatic fiber bundles are an example. The interactions are assumed to be weak, in the sense that the stress perturbation from a broken cell is much smaller than the mean stress in the system. The system starts intact with a surface-energy threshold required to break any cell sampled from an uncorrelated quenched-disorder distribution. The evolution of this heterogeneous system is ruled by Griffith's principle which states that a cell breaks when the release in potential (elastic) energy in the system exceeds the surfaceenergy barrier necessary to break the cell. By direct integration over all possible realizations of the quenched disorder, we obtain the probability distribution of each damage configuration at any level of the imposed external deformation. We demonstrate an isomorphism between the distributions so obtained and standard generalized Ising models, in which the coupling constants and effective temperature in the Ising model are functions of the nature of the quenched-disorder distribution and the extent of accumulated damage. In particular, we show that damage models with global load sharing are isomorphic to standard percolation theory and that damage models with a local load sharing rule are isomorphic to the standard Ising model, and draw consequences thereof for the universality class and behavior of the autocorrelation length of the breakdown transitions corresponding to these models. We also treat damage models having more general power-law interactions, and classify the breakdown process as a function of the power-law interaction exponent. Last, we also show that the probability distribution over configurations is a maximum of Shannon's entropy under some specific constraints related to the energetic balance of the fracture process, which firmly relates this type of quenched-disorder based damage model to standard statistical mechanics.
\end{abstract}

DOI: 10.1103/PhysRevE.71.046127 PACS number(s): 46.50.+a, 46.65.+g, 62.20.Mk, 64.60.Fr

\section{INTRODUCTION}

The physics of breakdown processes that lead, for example, to stress-induced catastrophic failure of both manmade and geological structures, remains an ongoing subject of research. Stress-induced fracture of a homogeneous material containing a geometrically simple single flaw has been studied since the work of Griffith [1] and is now well understood. However, the breakdown of heterogeneous structures, in which the local mechanical properties are randomly distributed in space and/or time, continues to present challenges despite the many advances over the last 15 years [2]. The difficulty is in characterizing and quantifying the effects of interaction between the multitude of constituents.

Most of the knowledge about these types of systems has been obtained from lattice network simulations. One of the most well-studied lattice network models is the 80 year old fiber bundle model (FBM) [3-6] that describes the rupture of bundles of parallel fibers. This model originally considered elastic fibers of identical elastic constant, breaking when their elongation exceeds individual thresholds distributed according to a given uncorrelated random distribution. A global

\footnotetext{
*Electronic address: Renaud.Toussaint@fys.uio.no; URL: http:// folk.uio.no/renaud/

${ }^{\dagger}$ Electronic address: srpride@lbl.gov
}

load sharing rule (GLS) is assumed by which the load carried by a fiber is uniformly distributed to the surviving fibers when it breaks. Analytical results for this model have been obtained [7-11] that concern the average load-deformation properties [7-9], the distribution of avalanches [10], or the relationship between such quenched-disorder based models and standard statistical mechanics [11].

The original FBM model with global load sharing has been generalized to allow for nonuniform load sharing rules, considered either as purely local load sharing (nearestneighbor interactions), in which case distribution of avalanches and mechanical properties have been analytically studied [12-14], or as power laws of distance from the failed fiber, which have been studied numerically $[15,16]$.

Closely related to these models of fiber bundles in elastic interaction, many studies have addressed fuse networks [17-25], or networks of isotropic damage with interactions having the range of the elastostatic Green function [26-28].

The classification of lattice breakdown processes as critical-point phenomena is still subject to debate [9,29-37]. Local load sharing models are usually understood as breaking through a process similar to a first-order transition [29], while models with long-range elastic interactions or GLS are analyzed either as a critical-point transition [9,29,30,35], or as a spinodal nucleation process $[31,38]$. The issue depends on whether the accumulated damage has a correlation length that diverges as a power law of the average deformation in the approach to macroscopic failure. Numerical evidence in 
the literature suggests that the nature of the correlation length in the approach to failure depends on the specific model being analyzed, the damage interaction range, and the type of quenched-disorder distribution considered. A major difficulty of such attempts to classify the breakdown process is that no analytical form for the distribution of the damage configurations at a given external load is available (at least that has a firm basis). Numerical simulations of the scaling behavior of avalanches are limited in size due to computational restrictions which makes critical-point analysis difficult.

It is proven here that quasistatic interacting damage models such as the FBM, that possess randomly quenched local breaking thresholds, can be mapped onto percolation, Ising, or generalized Ising models depending on the range of the damage interaction. Our demonstration holds in the limit of small damage; i.e., under the restriction that the stress perturbation emanating from each broken cell is much smaller than the average stress in the system. More specifically, the change in elastic energy in the system at fixed imposed boundary deformation due to damage that arrives is the sum of a mean-field softening term proportional to the number of damaged cells, and an additive function over pairs of damaged cells that is small compared to the mean-field softening terms. This corresponds to the general validity conditions of a Born approximation. In real solids accumulating damage, the cracks represent only small local changes in the elastic moduli, and so this weak perturbation limit has direct practical importance.

The stress-induced emergent-damage distribution is proven to be a Boltzmannian with a temperature (probabilistic energy scale) that is an explicit analytical function of both the applied deformation and the quenched-disorder threshold probability distribution funtion (PDF). The damage-model Hamiltonian is shown to have an Ising form with coupling constants that have explicit functional dependence on the applied deformation, the quenched-disorder PDF, and the nature of the damage interaction (nearest neighbor or long range). The critical point of the Ising model is of course well known. So for a given numerical simulation or laboratory experiment, one can use the expressions for the coupling constants developed here to determine how the system is evolving in coupling-parameter space as deformation increases and thus be able to predict the nature of any phase transition that might arrive (first order, continuous, or no transition). Knowing whether catastrophic failure (i.e., damage correlation-length divergence) is an imminent possibility has practical application.

We have recently proposed a statistical theory for the localization of oriented fractures that emerge and elastically interact when the system has a shear stress applied to it [39-41]. The distribution of the emergent crack states was obtained using the postulate that the fracture arrival would maximize Shannon's entropy under constraints representing the energetic balance of the process. In the present work, we do not make this postulate but instead prove its validity by direct integration over the damage evolution. The present work also presents more general ranges of interaction. However, unlike our previous work, the present analysis is for a purely isotropic (scalar) description of damage interaction. The interaction of real fractures in an anisotropic load, where microfractures present high aspect ratios, necessarily requires a tensorial elastic description as in our earlier work [39-41].

The organization of the paper is as follows. In Sec. II, we introduce (and justify in the Appendix) the general type of scalar damage models that are considered. In Sec. III, the probability of each damage configuration is obtained by integrating over all paths that lead to it. In Sec. IV, we establish the relationship between these configurational distributions and standard statistical mechanics, which allows the standard toolbox of statistical mechanics to be applied to damage models entirely based on quenched disorder. These analytical developments will then be utilized in Sec. V to establish that fracture processes in such damage models are isomorphic to percolation for GLS (Sec. V A), or to the Ising model for nearest-neighbor interactions (Sec. V B). This allows us to isolate some transition points in these models, and to predict the nature of the correlation length in the approach to the transition. We will also discuss in Sec. V C the case of damage models with arbitrary power-law decay of the interactions, and show how they are related to generalized Ising models, which can themselves be mapped onto standard ones via renormalization of the coupling constants. The results are summarized and discussed in a concluding section.

\section{DEFINITION OF THE DAMAGE MODELS CONSIDERED}

Our models reside on a regular lattice of dimension $D$ (e.g., a square lattice in $D=2$ ). Each cell of the lattice has a location $x$ within the set $\Omega$ of cells making up the lattice, and has a state described by a local order parameter $\varphi_{x}$, where $\varphi_{x}=0$ if the cell is intact and $\varphi_{x}=1$ if it is broken. There is a local stress and strain associated with each cell. The cells elastically interact with each other; however, such interaction must be isotropic for the present analysis to apply. A configuration of damage is described as a damage field $\varphi$ corresponding to the set of $N$ local variables $\varphi \equiv\left\{\varphi_{x}\right\}_{x \in \Omega}$ where $N=N(\Omega)$ is the total number of cells in the system.

Our systems are initially uniform by hypothesis; i.e., they have a homogeneous damage field $\varphi=0$ at zero strain and stress and each cell starts with the same elastic moduli. Strain is progressively applied through the application of a uniform normal displacement $l$ at the edges of the system. A cell breaks at constant applied $l$ when the energy required to break it (which is a random quenched threshold sampled from a probability distribution function) just equals the reduction in stored elastic energy in the lattice due to the break.

A key requirement of the models treated here is that the stress perturbation emanating from a broken cell must be weak enough that a first Born approximation holds; i.e., stress interaction between any two broken cells is allowed for while simultaneous interaction between three or more broken cells is not. This approximation is valid whenever the stress perturbation due to a broken site is much smaller than the mean stress in the system.

One specific realization of such a "weak damage" model is an appropriately defined fiber bundle model. In the model, a set of $N$ elastic fibers are stretched between a free rigid 
plate and an elastic half space. The rigid plate is displaced by a controlled amount $l$ that puts the fibers in tension. Once fibers begin to break, elastic interactions occur through the elastic solid. As demonstrated in the Appendix, such interactions will be weak if either (1) the elastic solid is much stiffer than the fiber material, or (2) the fibers are much longer than wide and are sufficiently widely placed. In the limit that the elastic half space becomes rigid, this model reduces to the classic global load sharing fiber bundle.

Another realization is a uniform isotropic solid divided into $N$ cells. Uniform displacements $l$ are applied to the limiting faces of the lattice in such a way that the material is in a state of uniform dilation. The damage that arrives is assumed to change the isotropic moduli without creating anisotropy in the process. For example, the damage might be modeled as a spherical cavity that opens at the center of the cell thus reducing the elastic moduli of that cell. The weak interaction is guaranteed if the change in the cell modulus is small. We emphasize that the assumption of weak damage holds for any configuration of damage over the underlying lattice, including damage states with large fractions of broken cells.

As demonstrated in the Appendix, the energy $E_{p}$ reversibly stored in such systems when the system is in a damage state $\varphi$ at the applied loading level $l$ is

$$
\begin{gathered}
E_{p}[\varphi, l]=\left(C_{0}+C_{1}+C_{2}\right) l^{2}, \\
C_{0}=N, \\
C_{1}=-c \sum_{x \in \Omega} \varphi_{x}, \\
C_{2}=-\varepsilon \sum_{x, y} J_{x y} \varphi_{x} \varphi_{y},
\end{gathered}
$$

where $c$ is a positive constant in the range $0<c \leqslant 1$ that is independent of the damage or deformation state, $\varepsilon$ is a small positive parameter in the range $0<\varepsilon / c \ll 1$ that controls the strength of the stress perturbations, and $J_{x y}$ are $O(1)$ coupling constants that allow for the load redistribution between cells at positions $x$ and $y$ when a cell is broken. Various spatial ranges for $J_{x y}$ are considered including (1) global load sharing in which case $J_{x y}=0$; (2) local load sharing in which case $J_{x y}=\alpha>0$ is a constant of order unity for pairs $x y$ of nearest neighbors, 0 otherwise; (3) elastic load sharing in which case $J_{x y} \sim(\ell /|x-y|)^{D}$ where $\ell$ is the lattice step size; and (4) fiber-bundle elastic sharing with fibers interacting through an elastic plate in which case $D=2$ and $J_{x y} \sim \ell /|x-y|$ (see the Appendix).

The local load sharing case (2) can in principle happen in a fiber bundle stretched between plates enduring both elastic and plastic deformations capable of screening the stress perturbations caused by broken fibers to only nearest neighbors, which always can carry some load if damaging them only decreases their elastic constant (i.e., $c<1$ ). We treat case (2) for the sake of generality and do not specify the detailed constitutive relations required for it to be realized in practice.

The cost in surface energy to break any of the cells (which represents either the energy required to create new surface area within a cell or to break a fiber) is a random variable fixed $a b$ initio, with no spatial correlations between the different cells. The breaking energy is thus a quenched uncorrelated disorder, described by a probability distribution function $p(e)$ for which $p(e) d e$ is the probability that a cell's surface energy is in $[e, e+d e]$, and having a cumulative distribution $P(e)=\int_{0}^{e} p(z) d z$. For a given realization of each cell's surface energy $e_{x}$, there is thus a certain total surface energy

$$
E_{s}[\varphi]=\sum_{x \in \Omega} e_{x}\left(1-\varphi_{x}\right)
$$

associated with each damage field $\varphi=\left\{\varphi_{x}\right\}_{x \in \Omega}$.

Given that $B \subset \Omega$ is a certain subset of locations, the notation $\varphi^{B}$ refers to the state where this subset is broken and its complementary intact; i.e., $\varphi_{x}^{B}=1$ for every $x \in B$, and $\varphi_{x}^{I}=0$ for every $x \in I=B^{C}$.

As the external deformation $l$ is increased, damage evolution is ruled by Griffith's principle: Given the system in a certain configuration $\varphi^{B}$ and deformation $l$, it can undergo a transition towards a more broken state $\varphi^{B \cup\{x\}}$ that differs from the previous state by one additional broken cell at $x$, if the release in potential energy is equal to the surface energy cost of the new break; i.e., if

$$
\Delta E_{p}\left[\varphi^{B}, x, l\right]=e_{x},
$$

where

$$
\Delta E_{p}\left[\varphi^{B}, x, l\right]=E_{p}\left[\varphi^{B}, l\right]-E_{p}\left[\varphi^{B \cup\{x\}}, l\right]=\left(c+\varepsilon \sum_{y} J_{x y} \varphi_{y}\right) l^{2} .
$$

If $e_{x}>\Delta E_{p}\left[\varphi^{B}, x, l\right]$ for any surviving cell $x \in I$, there is no break and the deformation can be further increased while the system remains in the same state $\varphi^{B}$. If a break happens in cell $x$, which leads to the new state $\varphi^{E}$ where $E=B \cup\{x\}$ while the external deformation $l$ is kept constant, there is a possibility of avalanche at fixed $l$ if there is some $y \in E^{C}$ such that

$$
\Delta E_{p}\left[\varphi^{E}, y, l\right] \geqslant e_{y} .
$$

If there is more than one possible location satisfying Eq. (8), the one that breaks is determined by maximizing the energy release; i.e., its location corresponds to

$$
\Delta E_{p}\left[\varphi^{E}, y, l\right]-e_{y}=\max _{z \in E^{C}}\left(\Delta E_{p}\left[\varphi^{E}, z, l\right]-e_{z}\right) .
$$

The avalanche test [Eq. (8)] is then computed again until the system stabilizes in a given configuration.

Eventually, although we have chosen to base the evolution of our damage model on minimization of energy, we note that the formal integration of path probabilities presented in the following sections could similarly be obtained as well for the case of a rule based on force thresholds, with at any given external deformation a force carried per intact fiber equal to an average one, plus perturbations due to the already broken fibers. However, this approach will not be pursued here. 


\section{PROBABILITY DISTRIBUTION OF DAMAGE STATES}

The probability of occurrence of any damage configuration $\varphi$ is now determined when the system is at a given external deformation $l$ that was reached monotonically ( $l$ as defined here does not include any elastic unloading).

More specifically, in the previous section, we have defined a deterministic rule for the evolution of our studied systems. Given a particular realization of the quenched disorder (QD) over each lattice cell, and an initially intact state, the system experiences a progressive damage that builds up as the external deformation is increased. Given a specific emergent damage configuration over the lattice, and a final elongation $l$, we determine here what fraction of all possible realizations of the QD lead to the given damage state at the given deformation. By definition, this probability of occurrence integrates over all possible ways (and orders) through which this configuration arises at final $l$. Consequently, the probability distribution over configurations that we obtain contains no information about the order at which the cells broke.

The PDF we obtain thus corresponds to the classical quantity estimated in numerical simulations of deterministic systems with QD in the damage thresholds; namely, given $N_{r}$ realizations of the QD, a specific damage configuration is realized $n_{r}$ times, and the probability of occurrence of this configuration is estimated as the fraction $n_{r} / N_{r}$ at large $N_{r}$.

We will first summarize the results for the simplest case, global load sharing, which was performed in [11], and which will serve as a basis for a perturbative treatment to include the effect of interactions.

\section{A. Global load sharing}

In this case, the interaction term of Eq. (4) is $C_{2}=0$ for any configuration, and from Eq. (7), $\Delta E_{p}[\varphi, x, l]=c l^{2}$ regardless of the state $\varphi$ and new break location $x$ considered.

Each of the cells then shares the same level of deformation $l$, and the probability for any one of them to be broken is simply (from the cumulative surface energy distribution)

$$
P_{0}=P\left(e<c l^{2}\right)=\int_{0}^{c l^{2}} p(z) d z,
$$

independently of the state of the other cells since there are no interactions between them, and each fiber simply sustains the mean-field deformation $l$, which was reached monotonically from zero.

The probability of being in a given configuration $\varphi$ with $n$ cells broken out of $N$ is then

$$
P[\varphi, l]=P_{0}(l)^{n}\left[1-P_{0}(l)\right]^{N-n} .
$$

This corresponds to the behavior of a so-called "two-state system" in which each of the $N$ independent sites has a probability $P_{0}(l)$ of being broken and $1-P_{0}(l)$ of being intact.

To avoid confusion, note that the above probability is the probability of reaching a specific configuration $\varphi$ at $l$, which specifies the state of every cell, and depends only on the number of broken cells $n$ in the state $\varphi$ in this GLS case. Accordingly, the probability $P[n, l]$ of reaching any state with $n$ broken cells can be obtained by adding this probability $P[\varphi, l]$ for every state $\varphi$ containing $n$ broken cells out of $N$, so that $P[n, l]=N ! /[(N-n) ! n !] P_{0}(l)^{n}\left[1-P_{0}(l)\right]^{N-n}$.

The consequences of this distribution function for the mechanical behavior and correlation length at the transition point of macroscopic rupture will be developed in Sec. V A As a consistency check, we will then show that the derived ensemble-averaged mechanical response of the system has exactly the known form [7] for a fiber bundle in global load sharing, in the limit of large sizes $N$.

Note that working at an imposed deformation level $l$, rather than an imposed total force $\tau$ over the bundle, results in the local deformation level of each cell being that imposed on the entire system. Considering instead an imposed total force $\tau$ carried by the bundle would require global information on the total number of broken cells $n$ in order to know the elongation of each cell (and hence the force carried by each surviving cell). In the present notation, the force carried by an intact cell is $d E / d l=2 l$, the one carried by a damaged cell is similarly $2(1-c) l$, so that the total force carried by the bundle is $\tau=(N-n c) 2 l$. If we impose the total force $\tau$ on the bundle as an external control parameter, the probability for any cell to break would depend both on $\tau$ and on the past history of the bundle, through the dependence of the individual elongations (or forces) on the number $n$ of already broken cells, since $l=\tau /[2(N-n c)]$. Considering the imposed total force $\tau$ as a control parameter allows us to compute the statistics of avalanches $[10,12,13]$, but involves more complicated expressions to obtain the probability of states at a fixed global force level [7]. This led us to choose here to work instead at imposed global deformation $l$, which immediately makes the states of the cells at given elongation independent random variables from each other in the GLS case. The advantage of this choice in the case of interactions of variable ranges, where these events are no longer statistically independent, is that we will be able to analytically explore the effect of these interactions by a perturbative treatement from the simple Eq. (11) above.

\section{B. Local load sharing}

\section{Damage-state probability distribution}

We now consider the case where each broken cell increases the local deformation by an amount $\alpha \varepsilon l$ on each of its nearest neighbors; i.e., $J_{x y}=\alpha$ for each nearest-neighbor pair, or $J_{x y}=0$ for more distant cells.

Note that for a given cell $x$, the potential energy release defined in Eq. (7), $\Delta E_{p}[\varphi, x, l]$, is a growing function of both the deformation $l$ and the subset of cracked cells $\varphi$ considered; i.e., if we consider two subsets $B \subset D$ and $x \in D^{C}$, then $\Delta E_{p}\left[\varphi^{D}, x, l\right] \geqslant \Delta E_{p}\left[\varphi^{B}, x, l\right]$. Physically, this inequality means that the local load over each cell increases with the external load imposed, and that any cell breaking anywhere else induces an additional increase in local load. This inequality will play a key role in obtaining the damage-state probability distribution.

To aid the pedagogic development, we first derive the probability of occurrence of a configuration $\varphi^{B}$ with $n$ iso- 
lated broken cells forming a subset $B$, which do not share any common nearest neighbors. There are $z n$ nearest neighbors to these $n$ cells (in a subset $F$ corresponding to the boundary of broken cells) where $z$ is the coordination number of the lattice considered. There are then $N-(z+1) n$ cells completely isolated from the broken ones in the subset $(B \cup F)^{C}$. For a cell $x$ having broken from an intermediate stage $\varphi^{A}$ with $A \subset B$, at an intermediate external load $h$ $\in[0, l]$, the change in the stored energy satisfies $\Delta E_{p}\left[\varphi^{A}, x, h\right]=c h^{2} \leqslant c l^{2}$. For every cell having survived, we have either $\Delta E_{p}\left[\varphi^{B}, x, l\right]=(c+\alpha \varepsilon) l^{2}$ if $x \in F$ ( $x$ is on the boundary of the broken cell set), or $\Delta E_{p}\left[\varphi^{B}, x, l\right]=c l^{2}$ if $x$ $\in(B \cup F)^{C}$ ( $x$ is completely disconnected from the broken cell set). Applying Griffith's principle [Eq. (6)] to every cell and intermediate deformation $h \in[0, l]$, and using the monotony of $\Delta E_{p}[\varphi, x, l]$ in both $l$ and $\varphi$, we obtain that a necessary and sufficient condition for all cells in $B$ to be broken is that their surface-energy thresholds were below $c l^{2}$, while those of their neighbors in $F$ were above $(c+\alpha \varepsilon) l^{2}$, and the remaining ones in $(B \cup F)^{C}$ above $c l^{2}$. Defining

$$
P_{1}(l)=P\left(e<(c+\alpha \varepsilon) l^{2}\right)=\int_{0}^{(c+\alpha \varepsilon) l^{2}} p(z) d z,
$$

this then implies that

$$
P[\varphi, x]=P_{0}^{n}\left(1-P_{1}\right)^{z n}\left(1-P_{0}\right)^{N-(z+1) n}
$$

is the probability of occurrence of such a configuration.

We now pass to the more general case. In the argument, we obtain upper and lower bounds for the probability of some arbitrary damage state, and then demonstrate that in the limit $\varepsilon / c \ll 1$, the two bounds converge to the unique probability distribution of interest.

For any configuration $\varphi, u_{k}$ is defined as the number of intact cells with $k$ broken nearest neighbors and $n_{k}$ as the number of broken cells with $k$ broken neighbors. If $n$ out of the $N$ cells are broken, we have $\Sigma_{k} n_{k}=n$ and $\Sigma_{k} u_{k}=N-n$. The way the above quantities are associated with any particular configuration is illustrated in Fig. 1.

For a cell $x$ that broke from an intermediate state $\psi \subset \varphi$ with $k$ already broken neighbors in $\psi$, at an external load $h$, we have

$$
\Delta E_{p}[\psi, x, h]=(c+k \alpha \varepsilon) h^{2} .
$$

Using again the monotony of $\Delta E_{p}$, a necessary and sufficient condition for any intact cell $y \in B^{C}$ to have survived is that its threshold exceeded $\Delta E_{p}[\varphi, y, l]$. The probability for each of these independent statistical events to occur is expressed as $1-P_{k}(l)$, where

$$
P_{k}(l)=P\left(e<(c+k \alpha \varepsilon) l^{2}\right) .
$$

For any cell that broke $x \in B$, we note that they have broken with certainty at the ultimate deformation $l$ if the external load was sufficient to trigger their break without the help of overload due to breaks of the other ones; i.e., they have broken with certainty if their energy threshold was below $\Delta E_{p}\left(\varphi^{\emptyset}, x, l\right)=c l^{2}$ where $\emptyset$ denotes the empty set (no broken cells). Furthermore, if every threshold in $B$ was below $c l^{2}$,

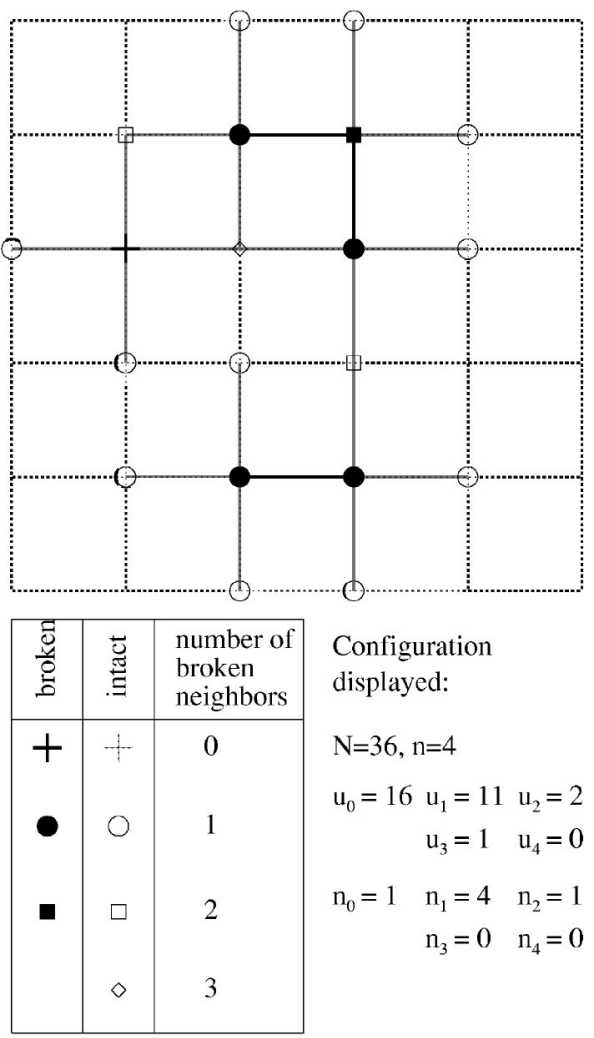

FIG. 1. Typical configuration and associated connectivity measures.

except a particular one $x \in B$ which has $k_{x}$ broken neighbors in the considered configuration $\varphi^{B}$ and has a breaking energy between $c l^{2}$ and $\left(c+k_{x} \alpha \varepsilon\right) l^{2}$, the $k_{x}$ neighbors of this considered cell $x$ will have broken with certainty at the ultimate load $l$, so that $x$ will also break with certainty under the effect of the overload due to its broken neighbors. The probability that this individual threshold broke under the sole effect of the overload due to its neighbors can be expressed

$$
\Delta P_{k_{x}}=P_{k_{x}}(l)-P_{0}(l) .
$$

Thus, a lower bound for the probability of occurrence of the configuration $\varphi^{B}$ can be expressed as

$$
P\left[\varphi^{B}, l\right]>\left[P_{0}^{n}+\left(\sum_{x \in B} P_{0}^{n-1} \Delta P_{k_{x}}\right)\right] \prod_{k \in N}\left(1-P_{k}\right)^{u_{k}},
$$

where the index $k$ runs formally to $N$; however, $u_{k}=0$ when $k>z$ (the coordination number of the lattice).

For any cell $x \in B$ that broke, its associated energy threshold was necessarily lower than $\Delta E_{p}\left[\varphi^{B \backslash\{x\}}, x, l\right]$ where $B \backslash\{x\}$ denotes the set $B$ with cell $\{x\}$ excluded from it. Thus, an upper bound for the probability of occurrence of the configuration under study is

$$
\begin{aligned}
P\left[\varphi^{B}, l\right] & <\left[\prod_{x \in B} P_{k_{x}} \prod_{k}\left(1-P_{k}\right)^{u_{k}}\right. \\
& =\left[\prod_{x \in B}\left(P_{0}+\Delta P_{k_{x}}\right)\right] \prod_{k}\left(1-P_{k}\right)^{u_{k}} .
\end{aligned}
$$

For a continuous PDF over thresholds (which guarantees no 
jumps in the cumulative distribution $P$ and is the only restriction placed on the PDF), $\Delta P_{k}$ is a quantity of order $\varepsilon$, and $P_{0}$ is of order 1. In the limit of a Born model (weak stress perturbations for which $\varepsilon / c \ll 1$ ), the upper and lower bounds for the probability of occurrence are identical to order $\varepsilon$. We have thus established in this framework that

$$
P[\varphi, l]=\prod_{m=0}^{\infty} P_{m}{ }_{m} \prod_{k=0}^{\infty}\left(1-P_{k}\right)^{u_{k}} .
$$

\section{Identification of a surface tension and cohesion energy}

The above can be reexpressed for small interactions $\varepsilon$ $\ll 1$ by a Taylor expansion of the cumulative QD distribution as

$$
\Delta P_{k}(l)=\gamma(l) k, \quad \text { where } \gamma=p\left(c l^{2}\right) \alpha \varepsilon l^{2} .
$$

Then, Eq. (19) becomes

$$
\begin{aligned}
\ln P[\varphi, l]= & \ln \left[\prod_{m=0}^{\infty}\left(P_{0}+\gamma m\right)^{n_{m}} \prod_{k=0}^{\infty}\left(1-P_{0}-\gamma k\right)^{u_{k}}\right] \\
\simeq & n \ln P_{0}+(N-n) \ln \left(1-P_{0}\right) \\
& +\frac{\gamma}{P_{0}} \sum_{m=1}^{\infty} m n_{m}-\frac{\gamma}{1-P_{0}} \sum_{k=1}^{\infty} k u_{k} \\
= & n \ln P_{0}+(N-n) \ln \left(1-P_{0}\right) \\
& +2 \frac{\gamma}{P_{0}} n_{I}-\frac{\gamma}{1-P_{0}} n_{S},
\end{aligned}
$$

where in the state $\varphi$ considered, $n$ is the number of broken sites, and $n_{I}$ and $n_{S}$ refer, respectively, to the number of internal bonds between pairs of broken cells and the number of boundary bonds between broken and intact cells.

These probabilities are thus of the form

$$
P[\varphi, l]=P\left[\varphi^{\emptyset}\right] e^{n \ln \left[P_{0} /\left(1-P_{0}\right)\right]+2 n_{I} \gamma / P_{0}-n_{S} \gamma /\left(1-P_{0}\right)}
$$

with the probability of the intact state

$$
P\left[\varphi^{\emptyset}\right]=\left(1-P_{0}\right)^{N} .
$$

The probabilities are thus classic Boltzmann distributions $P\left[\varphi^{\emptyset}\right] e^{-H}$ where

$$
-H[\varphi, l]=\ln \left[\frac{P_{0}}{\left(1-P_{0}\right)}\right] n+2 \frac{\gamma}{P_{0}} n_{I}-\frac{\gamma}{1-P_{0}} n_{S} .
$$

For no interactions, $\gamma=0$ and the above reduces to the global load model of Eq. (11). Since $n$ and $n_{I}$ can be formally interpreted as volume integrals over the interior of clusters of broken cells, and $n_{S}$ as a surface integral along the boundary of these clusters, we can make the following analogies to the quantities of classical statistical physics:

$$
\begin{gathered}
\ln \left[P_{0} /\left(1-P_{0}\right)\right] \rightarrow-\mu / T \quad \text { (chemical potential), } \\
2 \frac{\gamma}{P_{0}} \rightarrow \frac{-e}{T} \quad \text { (bulk energy), }
\end{gathered}
$$

$$
\frac{\gamma \ell}{1-P_{0}} \rightarrow \frac{\gamma_{S}}{T} \quad \text { (surface tension), }
$$

where $\ell$ is the lattice step size. The first term in Eq. (25) accounts for the average energy required to break a cell, the second term for an increase in the probability of finding some clusters of connected cracks due to positive interactions between them, and the third term for a decrease in the probability of finding clusters with a long interface between cracked and noncracked regions due to the fact that intact cells along the boundary are more likely to have broken from overloading from cracked neighbors, thus leading to even more fractured states.

In the beginning of the process, the two first terms dominate, and to leading order

$$
-H[\varphi, l]=\left(\ln P_{0}\right) n+2 \frac{\gamma}{P_{0}} n_{I} .
$$

\section{Arbitrary-range interactions}

As long as screening effects are absent or neglected (as they are in the present model of weak interactions), the above arguments based on Griffith's principle and the monotony of $\Delta E_{p}$ in $\varphi$ and $l$ extend directly to the case of arbitrary ranges of interactions.

At external deformation $l$, a cell $x$ (1) has broken with certainty if the external load alone could break it, i.e., if its associated surface energy is lower than $c l^{2}$; and (2) is intact with certainty if the external load plus the load perturbation due to the broken cells in the final configuration could not break it at final deformation, i.e., if its surface energy is higher than $\left(c+\varepsilon \sum_{y} J_{x y} \varphi_{y}^{B}\right) l^{2}$. Denoting

$$
\begin{gathered}
P_{0}=P\left(e<c l^{2}\right), \\
\Delta P_{x}=P\left(e<\left(c+\varepsilon \sum_{y} J_{x y} \varphi_{y}^{B}\right) l^{2}\right)-P\left(e<c l^{2}\right) \\
=p\left(c l^{2}\right) \varepsilon l^{2} \sum_{y} J_{x y} \varphi_{y}^{B},
\end{gathered}
$$

a necessary condition to end up at a certain configuration $\varphi^{B}$ at deformation $l$ is that all surviving cells $x \in B^{C}$ in that configuration have their threshold above $\left(c+\varepsilon \sum_{y} J_{x y} \varphi_{y}^{B}\right) l^{2}$, and all cells that broke $x \in B$ have their threshold below ( $c$ $\left.+\varepsilon \sum_{y} J_{x y} \varphi_{y}^{B}\right) l^{2}$. Thus,

$$
P\left[\varphi^{B}, l\right]<\prod_{x \in B}\left(P_{0}+\Delta P_{x}\right) \prod_{z \in B^{C}}\left(1-P_{0}-\Delta P_{z}\right)
$$

provides an upper bound for the probabilities in the case of arbitrary ranges of interaction.

Conversely, a sufficient condition to end up in this configuration is that all surviving cells have their threshold above $\left(c+\varepsilon \Sigma_{y} J_{x y} \varphi_{y}^{B}\right) l^{2}$, and that the broken cells either (1) have all their thresholds below $c l^{2}$; or (2) all but one located at $x$ have such thresholds, the last one breaking only due to the overload from the others; i.e., the last one has its threshold bounded by $c l^{2}<e<\left(c+\varepsilon \sum_{y} J_{x y} \varphi_{y}^{B}\right) l^{2}$. This gives a lower 
bound for the probability of the configuration $\varphi^{B}$,

$$
P\left[\varphi^{B}, l\right]>\sum_{x \in B}\left(P_{0}+\Delta P_{x}\right) \prod_{y \in B \backslash\{x\}} P_{0} \prod_{z \in B^{C}}\left(1-P_{0}-\Delta P_{z}\right) .
$$

As earlier, both lower and upper bounds coincide to order $\varepsilon$, so that upon Taylor expanding $\ln P[\varphi, l]$ to this order, we again obtain the Boltzmannian $P[\varphi, l]=P\left[\varphi^{\emptyset}\right] e^{-H[\varphi, l]}$ with an intact probability given again by $P\left[\varphi^{\emptyset}\right]=\left(1-P_{0}\right)^{N}$ and

$$
\begin{aligned}
-H[\varphi, l]= & \ln \left[P_{0} /\left(1-P_{0}\right)\right] n+\frac{p\left(c l^{2}\right) \varepsilon l^{2}}{P_{0}} \sum_{x y} J_{x y} \varphi_{x} \varphi_{y} \\
& -\frac{p\left(c l^{2}\right) \varepsilon l^{2}}{1-P_{0}} \sum J_{x y} \varphi_{x}\left(1-\varphi_{y}\right) .
\end{aligned}
$$

In the beginning of the process, the two first terms once again dominate and

$$
-H[\varphi, l]=\ln \left[P_{0}\right] n+\frac{p\left(c l^{2}\right) \varepsilon l^{2} \sum_{x y} J_{x y} \varphi_{x} \varphi_{y}}{P_{0}} .
$$

This expresses the equivalence between this most general weakly interacting damage model and an Ising model with generalized interaction rules.

\section{EQUIVALENCE WITH A MAXIMUM ENTROPY POSTULATE}

We now obtain these same probability distributions using the standard entropy maximization argument. The reason for doing this is to make explicit connection to usual thermostatistics and to define a partition function from which all thermodynamic properties of interest may be obtained through differentiation.

It is convenient to introduce the index $j$ to denote each possible damage configuration $\varphi$. We postulate that the probability distribution function over configurations $j$ maximizes Shannon's disorder [42]

$$
S=-\sum_{j} p_{j} \ln p_{j}
$$

subject to the constraints

$$
\sum_{j} p_{j} E_{j}=U, \quad \sum p_{j} n_{j}=n, \quad \sum_{j} p_{j}=1, \quad \forall j, \quad l_{j}=l,
$$

where $U$ is the total average energy that has been put into the system and $n$ is again the average number of broken cells. The validity of this maximization postulate will be directly demonstrated in what follows. However, independent of the formal demonstration, one can anticipate that Shannon entropy should be maximized since the initial quenched disorder in the breaking energies allows each possible damage configuration to be accessible. The constraints allow for the content of the Griffith principle and are what make certain emergent damage states more probable than others.

Throughout the remainder of the paper, $p_{j}$ denotes the probability of finding a configuration $j$ over all possible re- alizations of the qd, when the system has been brought to average deformation $l$ starting from an initially intact state. The term $n_{j}$ now refers to the total number of cracks in the configuration $j$, while $n$ is the statistical average of $n_{j}$. A few classical results can directly be derived from these assumptions:

$$
p_{j}=\frac{e^{-\beta\left(E_{j}-\mu n_{j}\right)}}{Z},
$$

$$
Z \equiv \sum_{j} e^{-\beta\left(E_{j}-\mu n_{j}\right)},
$$

where $\beta=\partial S /\left.\partial U\right|_{l, n}$ and $\beta \mu=-\partial S /\left.\partial n\right|_{l, U}$. From these one further has

$$
\begin{gathered}
G \equiv-\ln (Z) / \beta, \\
G=U-S / \beta-\mu n, \\
d G=\tau d l-S d(1 / \beta)-n d \mu, \\
d U=\tau d l+d S / \beta+\mu d n,
\end{gathered}
$$

where

$$
\tau=\sum_{j} p_{j} \tau_{j} \quad \text { and } \quad \tau_{j} \equiv \frac{d E_{j}}{d l} .
$$

The thermodynamic parameters $\beta$ and $\mu$ are obtained here by comparing Eq. (38) to the earlier probabilities obtained by direct integration over the microstate space.

The PDF over configurations is a maximum of Shannon's entropy under the above constraints, if and only if there are two constants $(\beta, \mu)$ such that

$$
H_{j}-\beta\left(E_{j}-\mu n_{j}\right)=\mathrm{const} \quad \forall j
$$

with $H_{j}$ given by Eq. (34). From Eqs. (1)-(4) and Eq. (34), we have

$$
\begin{gathered}
-H_{j}=\ln \left(\frac{P_{0}}{1-P_{0}}\right) n_{j}+\frac{p\left(c l^{2}\right) \varepsilon l^{2}}{P_{0}} \sum_{x y} J_{x y} \varphi_{x} \varphi_{y} \\
-\frac{p\left(c l^{2}\right) \varepsilon l^{2}}{1-P_{0}} \sum_{x y} J_{x y} \varphi_{x}\left(1-\varphi_{y}\right), \\
E_{j}=\left(N-c n_{j}-\varepsilon \sum_{x y} J_{x y} \varphi_{x} \varphi_{y}\right) l^{2}, \\
\sum_{x y} J_{x y} \varphi_{x}\left(1-\varphi_{y}\right)=-\sum_{x y} J_{x y} \varphi_{x} \varphi_{y}+n_{j} \sum_{r} J_{r},
\end{gathered}
$$

where translational invariance of $J_{x y}=J_{r=|\mathbf{y}-\mathbf{x}|}$ has been assumed. Equation (41) then requires

$$
\beta=\frac{p\left(c l^{2}\right)}{P_{0}\left(1-P_{0}\right)},
$$




$$
\mu=\frac{1}{\beta} \ln \left[\frac{P_{0}}{\left(1-P_{0}\right)}\right]-l^{2}\left(c+\varepsilon P_{0} \sum_{r} J_{r}\right) .
$$

Thus, the PDF over configurations indeed maximizes entropy [Eq. (36)] under the constraints of Eq. (37), with no unknowns. The inverse temperature and chemical potential depend on the deformation level through Eqs. (43) and (44). They are well defined analytical functions of $l$ and the model parameters considered, the qd distribution via $P_{0}$, and the interaction coupling $J_{r}$. So the usual machinery of equilibrium statistical mechanics [Eqs. (38) and (39)] is valid and can be used for any of our damage models.

The autocorrelation function $\left\langle\varphi_{x} \varphi_{y}\right\rangle$ can therefore be obtained in the standard way by (1) defining a new Hamiltonian $E_{j}^{\prime}=E_{j}+\Sigma_{x} Q_{x} \varphi_{x}$ that incorporates a coupling with a formal external field $Q_{x}$; (2) evaluating the associated generalized partition function $Z^{\prime}$; and (3) performing second-order derivatives with respect to the external field,

$$
\left\langle\varphi_{x} \varphi_{y}\right\rangle=\left.\frac{1}{\beta^{2} Z^{\prime}} \frac{\partial^{2} Z^{\prime}}{\partial Q_{x} \partial Q_{y}}\right|_{Q \equiv 0} .
$$

Second-order derivatives of the free energy $G$ with respect to $\beta$ and $\mu$ can also be directly related to variances of the number of broken cracks $n_{j}$ and the potential energy $E_{j}$, but these standard derivations are left to the reader.

Note that the coupling constants $J_{r}$ above are nonrandom functions of $r$. All randomness has been integrated over, giving rise to the Boltzmann distribution and the two statistical parameters $\beta$ and $\mu$. An alternative approach used by other authors on similar systems, namely, random fuse networks, would be to not integrate over the underlying quenched disorder, but to instead map the quasistatic evolving system onto a random field Ising model [43] with zero temperature. In this case, the underlying quenched disorder would manifest as randomness in the coupling parameters of some Hamiltonian function that must be minimized to obtain the realized configurations. This is an alternative approach quite distinct from the one used here. Our approach is the usual one of equilibrium statistical mechanics, in which the coupling constants are nonrandom functions, but there are many configurations in the neighborhood of the minimum of the Hamiltonian $H$ that are of statistical importance in determining the average properties of the system.

The energy yardstick $1 / \beta$ (temperature) that decides whether a given configuration is statistically significant behaves regularly throughout the damage process (at least for continuous QD distributions). In the case of a uniform QD on $[0,1]$, it will take the particularly simple form $1 / \beta$ $=P_{0}\left(1-P_{0}\right)$, starting from zero and going back to it, with a maximum when $P_{0}=1 / 2$. The chemical potential $\mu$ behaves regularly as well.

\section{APPLICATIONS}

\section{A. Global load model}

A consistency check of the results in Sec. IV is now performed for the case of the simple global load sharing model. From Eqs. (43) and (44) with $J_{r}=0$, we have

$$
\begin{gathered}
E_{j}=\left(N-c n_{j}\right) l^{2} \\
\beta\left(E_{j}-\mu n_{j}\right)=-\ln \left[\frac{P_{0}}{1-P_{0}}\right] n_{j}+\frac{p\left(c l^{2}\right)}{P_{0}\left(1-P_{0}\right)}\left[\left(N-c n_{j}\right) l^{2}\right. \\
\left.+c n_{j} l^{2}\right] \\
=\ln \left[\frac{P_{0}}{1-P_{0}}\right] n_{j}+f(l)
\end{gathered}
$$

Independently, we also have the direct result from Eq. (11)

$$
p_{j}=P_{0}^{n_{j}}\left(1-P_{0}\right)^{N-n_{j}}=P_{0} e^{-n_{j} \ln \left[P_{0} /\left(1-P_{0}\right)\right]} .
$$

Thus, the Boltzmann distribution Eq. (38) with temperature and chemical potential given by Eqs. (43) and (44) is indeed identical to the known solution Eq. (48), which confirms the validity of the expressions for $\beta$ and $\mu$.

It is also instructive to look at all terms in the first law Eq. (39) to see exactly what they represent. The values of the average quantities in this simplest model can be obtained using the lemma

$$
\sum_{j} P_{0}^{n_{j}}\left(1-P_{0}\right)^{N-n_{j}} n_{j}=N P_{0},
$$

which is demonstrated by applying the operator $x \partial / \partial x$ to the binomial theorem,

$$
(x+y)^{N}=\sum_{n=0}^{N} \frac{N !}{(N-n) ! n !} x^{n} y^{N-n}=\sum_{j} n_{j} x^{n_{j}} y^{N-n_{j}},
$$

and then taking $x=P_{0}$ and $y=1-P_{0}$. Using Eqs. (36), (37), (40), (46), and (48), one obtains

$$
\begin{gathered}
n=P_{0} N, \\
U=\sum_{j} P_{0}^{n_{j}}\left(1-P_{0}\right)^{N-n_{j}}\left(N-c n_{j}\right) l^{2}=N\left(1-c P_{0}\right) l^{2} \\
S=-N\left[P_{0} \ln P_{0}+\left(1-P_{0}\right) \ln \left(1-P_{0}\right)\right] \\
\tau_{j}=\frac{d E_{j}}{d l}=\left(N-c n_{j}\right) 2 l \\
\tau=N\left(1-c P_{0}\right) 2 l .
\end{gathered}
$$

Taking the derivatives of these quantities yields

$$
\begin{gathered}
d n=N d P_{0}, \\
d S=\ln \left[\frac{P_{0}}{1-P_{0}}\right] d P_{0}, \\
\mu d n=\left(\frac{P_{0}\left(1-P_{0}\right)}{p\left(c l^{2}\right)} \ln \left[\frac{P_{0}}{1-P_{0}}\right]-c l^{2}\right) N d P_{0}, \\
\frac{d S}{\beta}=-\frac{P_{0}\left(1-P_{0}\right)}{p\left(c l^{2}\right)} \ln \left[\frac{P_{0}}{1-P_{0}}\right] N d P_{0}, \\
\mu d n+\frac{d S}{\beta}=-c l^{2} N d P_{0},
\end{gathered}
$$




$$
\begin{aligned}
\tau d l & =N\left(1-c P_{0}\right) 2 l d l \\
\mu d n+\frac{d S}{\beta}+\tau d l & =N\left(1-c P_{0}\right) 2 l d l-c l^{2} N d P_{0} \\
& =d\left[N\left(1-c P_{0}\right) l^{2}\right]=d U
\end{aligned}
$$

which is a consistency check for the validity of the first law [Eq. (39)]. The average mechanical behavior of this model, as well as the evolution of entropy and formal temperature, are illustrated in Fig. 2 for flat QD distributions between 0 and $l_{\max }$ and for various values of $c$ (the parameter that controls the relative change in stiffness due to a cell breaking).

Most importantly for our present purposes, since the probabity $P_{0}$ of having a site broken in this model is independent of the configuration and site location, the global load sharing damage model is exactly equivalent to the percolation model with occupation probability $P_{0}(l)$. There is a critical-point phase transition in this model, when $P_{0}\left(l_{c}\right)$ $=1 / 2$, for which $S$ goes through a maximum $S=N \ln 2$. The correlation length diverges then as

$$
\xi \sim\left|P_{0}(l)-1 / 2\right|^{-\nu}
$$

with $\nu=4 / 3$ in dimension $D=2$ [44]. We have in general

$$
P_{0}(l)-P_{0}\left(l_{c}\right) \sim p\left(c l_{c}^{2}\right)\left(l-l_{c}\right)
$$

and thus

$$
\xi \sim\left|l-l_{c}\right|^{-4 / 3} .
$$

In pathological cases, special QD distributions satisfy $p\left(c l_{c}^{2}\right)=0$ so that $P_{0}(l)-P_{0}\left(l_{c}\right) \sim\left(l-l_{c}\right)^{\alpha}$ with $\alpha \neq 1$. This results in

$$
\xi \sim\left|l-l_{c}\right|^{-4 \alpha / 3} .
$$

Note that in Ref. [11], we have treated this model with $c$ $=1$, which did not change anything in the nature of the transition, but only changed the minimum stiffness associated with the most damaged configuration where $\tau_{\min }=2 N(1$ $-c) l$, and, consequently, the position or existence of a peak stress in the average mechanical response $\tau(l)$. This is seen in Fig. 2(b): the existence, and the position of a possible peakstress position relative to the percolation transition (black dot $l / l_{\max }=1 / \sqrt{2}$ ), depends on the particular model considered. However, the divergence of the correlation length and the associated critical-point nature of the percolation transition are insensitive to $c$.

The present approach does not allow a direct exploration of the avalanche distributions as the critical point is approached. This is because the probability distribution over configurations was obtained at each elongation $l$ by averaging over all realizations of the quenched disorder. To obtain directly a result on avalanches, on the contrary, correlations between successive elongations should be considered for each realization of the QD, and the average over the QD should only be considered afterward. Our damage model nonetheless behaves as a fiber bundle with GLS for which results about the avalanche distribution have been determined $[10,12,13]$.

\section{B. Local load model}

In this case, $J_{x y}=\alpha$ when $x, y$ are nearest neighbors, and zero otherwise. To make explicit connection to the Ising model in its usual form, we map the order parameter onto $\{-1,1\}$ by defining $\sigma=2 \varphi-1$. The probability distribution over configurations is then

$$
P[\sigma, l]=\exp \left[\beta\left(E \sum_{x} \sigma_{x}+J \sum_{\langle x y\rangle} \sigma_{x} \sigma_{y}\right)\right] / Z,
$$

where $\langle x y\rangle$ denotes a sum on nearest neighbors only. This is exactly the classical Ising model, with coupling constant and external field given by

$$
\begin{gathered}
\beta J=\frac{\alpha p\left(c l^{2}\right) \varepsilon l^{2}}{4 P_{0}\left(1-P_{0}\right)}, \\
\beta E=\frac{1}{2} \ln \left[\frac{P_{0}}{1-P_{0}}\right]+\frac{2 \alpha p\left(c l^{2}\right) \varepsilon l^{2}}{P_{0}} .
\end{gathered}
$$

The critical point of this model is at [45]

$$
\left(\beta J_{c}, \beta E\right)=\left(A_{c}, 0\right)
$$

with $A_{c}=\ln (1+\sqrt{2}) / 2$. The external field $\beta E$ starts at infinitely negative values, and ends up at infinitely positive ones. It evolves continuously and thus necessarily crosses $E=0$ at the $l_{c}$ satisfying, from Eq. (61),

$$
P_{0}\left(l_{c}\right)\left\{\ln \left[1-P_{0}\left(l_{c}\right)\right]-\ln \left[P_{0}\left(l_{c}\right)\right]\right\}=4 \alpha p\left(c l_{c}^{2}\right) \varepsilon l_{c}^{2} .
$$

The mean-field percolation result of $P_{0}\left(l_{c}\right)=1 / 2$ is thus recovered when the coupling vanishes $(\alpha=0)$, which is a consistency check.

More generally, for nonzero nearest coupling constants $\alpha$, the system will undergo a first-order transition if at $l_{c}$ satisfying Eq. (62) the formal inverse temperature satisfies

$$
\begin{aligned}
\beta\left(l_{c}\right) J & =\frac{\alpha p\left(c l_{c}^{2}\right) \varepsilon l_{c}^{2}}{4 P_{0}\left(l_{c}\right)\left[1-P_{0}\left(l_{c}\right)\right]} \\
& =\frac{\ln \left[1-P_{0}\left(l_{c}\right)\right]-\ln \left[P_{0}\left(l_{c}\right)\right]}{16\left[1-P_{0}\left(l_{c}\right)\right]}>A_{c} .
\end{aligned}
$$

Depending on the value of $\beta\left(l_{c}\right) J$, the system can display four types of behavior, which are schematically depicted in Fig. 3.

(i) For many QD distributions, the first value of $l_{c}$ satisfying Eq. (62) occurs for very small values of $P_{0}\left(l_{c}\right)$, which correspond to small values of $2 \alpha p\left(c l_{c}^{2}\right) \varepsilon l_{c}^{2}$, since $x \ln [(1$ $-x) / x] \rightarrow 0^{+} \quad$ when $\quad x \rightarrow 0 . \quad$ In this case, $\ln \{[1$ $\left.\left.-P_{0}\left(l_{c}\right)\right] / P_{0}\left(l_{c}\right)\right\} /\left[1-P_{0}\left(l_{c}\right)\right] \simeq-\ln \left[P_{0}\left(l_{c}\right)\right] \geqslant 1$, and the condition of Eq. (63) is satisfied. The system thus goes through a first-order phase transition at this $l_{c}$ and there is a discontinuous jump in the average number of broken cells and the average stress (which are related to first derivatives of the free energy with respect to $l$ or $\mu$, and are similar to the average number of spins up in an Ising model) [46]. The correlation length increases up to the transition but remains finite. All of this behavior for such local load models has been documented in the literature $[12,13,15]$. 

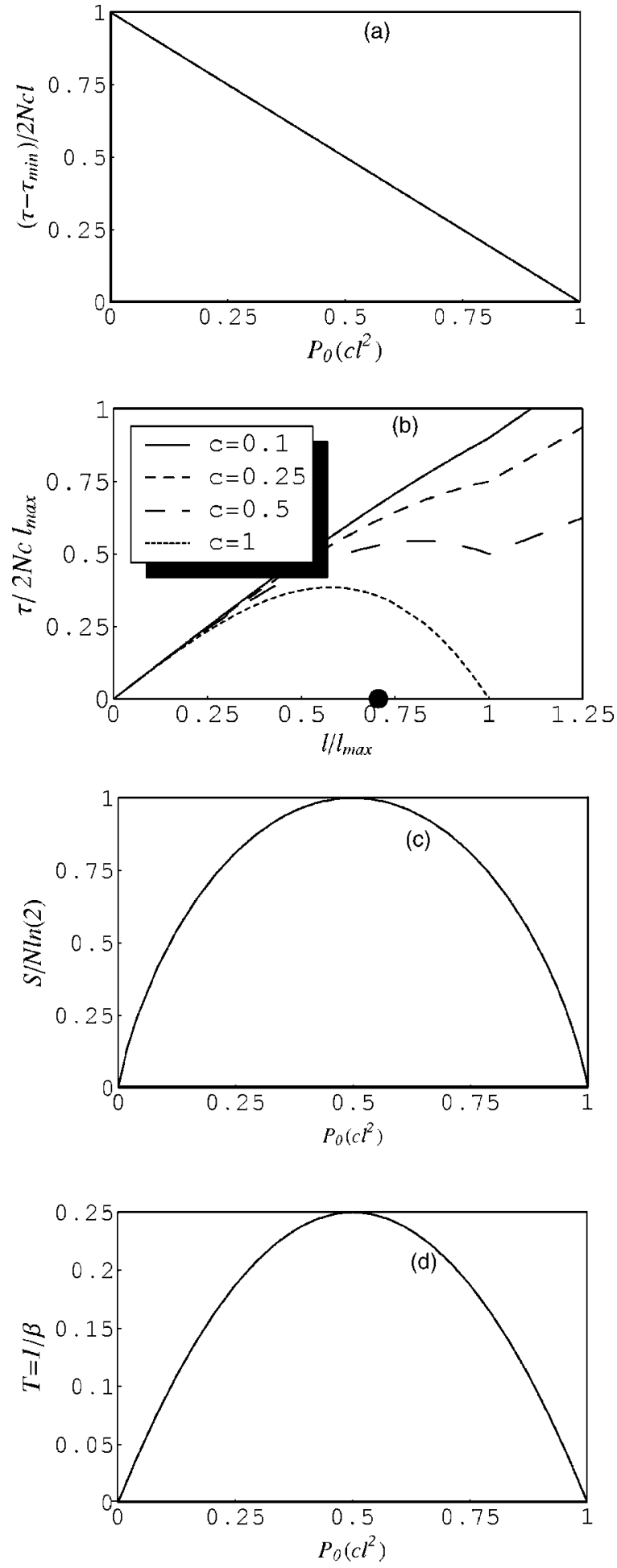

FIG. 2. Thermodynamic and mechanical response as a function of imposed deformation for the global load sharing model: (a) the difference between the average Young's modulus $\tau / l$ and the minimum Young's modulus $\tau_{\min } / l$ (which holds in the entirely damaged configuration); (b) average stress for a few particular models (the black dot is the percolation critical-point transition); (c) Shannon entropy; and (d) temperature.

However, three other behaviors are also possible if $\beta\left(l_{c}\right) J<A_{c}$ at the point $l_{c}$ at which the external field vanishes [Eq. (62)]. Whether this occurs is controlled by the type of

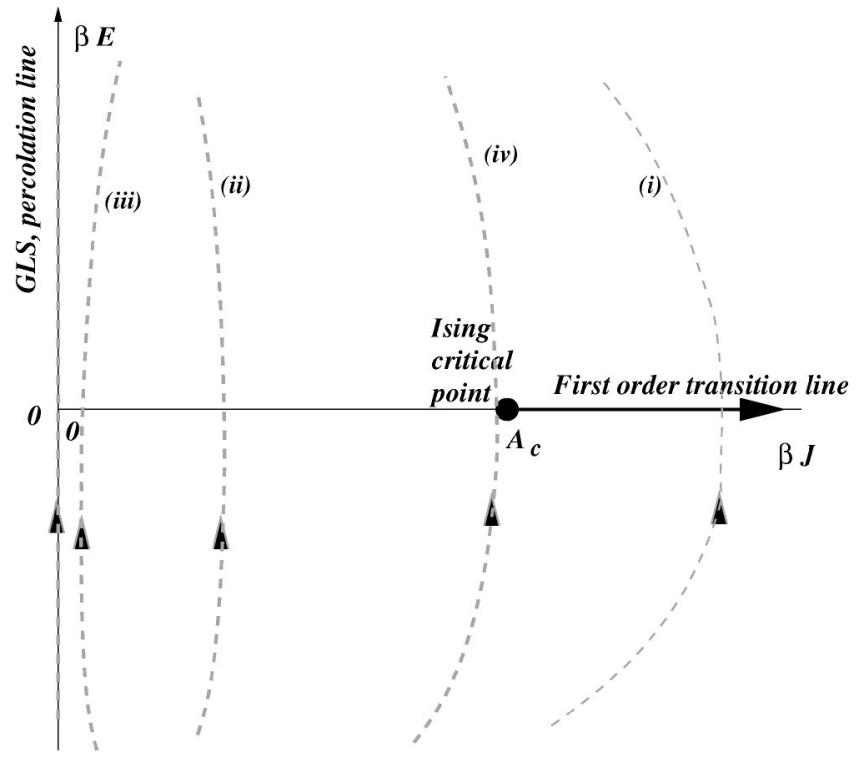

FIG. 3. Possible paths in the space of coupling constants of Ising models, under increasing imposed elongation associated with different local load sharing damage models.

QD distribution and the value of the coupling constant $\alpha$. When $\beta\left(l_{c}\right) J<A_{c}$, no first-order transition is encountered and one can further classify the transition into three subcategories.

(ii) If $\beta\left(l_{c}\right)$ has a finite value of order unity, significantly below $A_{c}$, the system simply goes continuously through $l_{c}$, without discontinuity in sustained load or average number of cracks. The correlation length remains finite.

(iii) If $\beta\left(l_{c}\right) J \ll 1$ (which should happen for vanishing $\alpha$ ), the distribution over configurations is dominated by the external field, and the system essentially behaves as a percolation model going through the percolation transition. Although there should be corrections due to the nonzero character of $\beta\left(l_{c}\right) J$, these might be smaller than the finite size corrections in numerical realizations, and the correlation length would then be found to diverge up to the system size as $\xi \sim\left|l-l_{c}\right|^{-4 / 3}$.

(iv) Finally, if $\beta\left(l_{c}\right) J \sim A_{c}^{-}$, the system comes close to the critical point of the Ising model at $l_{c}$, and the correlation length should diverge correspondingly as in an Ising system whose temperature comes close to $1 / A_{c}=T_{c}$ from above, while the external field changes sign. The slope of the average mechanical curve $\tau(l)$ should also locally diverge around $l_{c}$. The exponents associated with the divergence of $\xi$ as a function of $\left(l-l_{c}\right)$ depend on the way the critical point is approached as a function of $l$. For values of $l$ such that $\beta E(l) \ll 1$, we write $1 / \beta(l) J \sim T_{c}+f(l)$, and $\xi \sim\left|T(l)-T_{c}\right|^{-\nu}$ $=|f(l)|^{-\nu}$ where $\nu=1$ for the two dimensional (2D) Ising model. The correlation length therefore diverges as $\left|l-l_{c}\right|^{-\nu}$, unless the temperature has a quadratic minimum in $l$ close to $l_{c}$ in which case it diverges as $\left|l-l_{c}\right|^{-2 \nu}$.

\section{Power-law decay}

We last consider the general case of stress perturbations decaying as power laws of the distance to broken cells, 
$J(x) \sim \alpha(x / d)^{-\gamma} / \Phi(\gamma)$, where $\alpha$ is of order unity, $d$ is the lattice constant, and $\Phi(\gamma)=\sum_{y \neq x}(|y-x| / d)^{-\gamma}$ is a normalizing factor (which depends on the lattice size if $\gamma>D$, where $D$ is the system dimension). To be consistent, the models considered here are biperiodic of linear size $L$, and the interactions are put to 0 for distances above $L$. This type of model, considered for example by Hidalgo et al. $[15,16]$, allows us to span ranges between purely global sharing (when $\gamma=0$ ) to the local sharing limit $(\gamma \rightarrow \infty)$. Equations (42)-(44) show that this model leads to probability distributions over configurations of the form

$$
P[\sigma, l]=\exp \left[\beta\left(E \sum_{x} \sigma_{x}+\sum_{x \neq y} J(|x-y|) \sigma_{x} \sigma_{y}\right)\right] / Z,
$$

which is a generalized long-range Ising model with coupling constants and external field given by

$$
\begin{gathered}
\beta J(r)=\frac{\alpha p\left(c l^{2}\right) \varepsilon l^{2}}{4 P_{0}\left(1-P_{0}\right)} \frac{(r / d)^{-\gamma}}{\Phi(\gamma)}, \\
\beta E=\frac{1}{2} \ln \left(\frac{P_{0}}{1-P_{0}}\right)+\frac{\alpha p\left(c l^{2}\right) \varepsilon l^{2}}{2 P_{0}} .
\end{gathered}
$$

Note that although the case $\gamma \rightarrow \infty$ is isomorphic to the local load sharing model introduced in the previous section, the global load sharing model of Sec. V A corresponds to $\alpha=0$, but not to the exponent $\gamma=0$. The presence of the quadratic coupling makes this latter case isomorphic to a Curie-Weiss model, which is the mean-field theory of Ising models.

Such long-range Ising models are still an open area of research, with the precise value of the critical point for any power-law interaction not precisely known. Nonetheless, it has been proposed [46-48] that one can classify the criticality into two categories depending on whether $\gamma>D$ or $\gamma$ $<D$. In the shorter-range case, $\gamma>D$, this model admits a traditional thermodynamic limit when $L \rightarrow \infty$. Although $\Phi(\gamma)$ diverges when $L \rightarrow \infty$, the thermodynamic limit is well defined once $\Phi(\gamma)$ is introduced into the coupling constants $J(r)=(r / d)^{-\gamma} / \Phi(\gamma)$. Thus, the free or internal energy per lattice site, entropy, and magnetization all admit a finite limit when $L \rightarrow \infty$, and are functions of $\beta$ and the external field $E$. As $\gamma \rightarrow \infty$ in the thermodynamic limit, Eq. (65) reduces to the result of the standard Ising model [Eq. (60)] and so

$$
\lim _{\gamma \rightarrow \infty} \beta_{c}=\ln (1+\sqrt{2}) / 2 \text {. }
$$

It has also been shown [48] that as $\gamma \rightarrow D^{+}, \beta_{c} \sim 1$. Thus, for $\gamma / D$ in the range $\left[1^{+}, \infty\right], \beta_{c}$ is bound between 1 and $\ln (1$ $+\sqrt{2}) / 2$. However, more precise details of how $\beta_{c}$ varies with $\gamma$ in this range are not presently available.

In the other category of longer-range interactions where $\gamma / D$ lies in $\left[0,1^{-}\right]$, the only thing certain is that when $\gamma / D$ $=0$, the Curie-Weiss model is obtained for which $\beta_{c}$ is also of order unity. It has thus been conjectured that all power-law interactions for which $\gamma / D<1$ should be in the Curie-Weiss universality class [49]. The Curie-Weiss model corresponds to the mean-field coupling $J_{x y}=1 / N$, independently of $x$ and $y$, where $N$ is the total number of cells in the system.
For the Curie-Weiss model, the PDF over state configurations is $P[\sigma] \propto \exp \left[\beta\left(E \Sigma_{x} \sigma_{x}+\sum_{x y} \sigma_{x} \sigma_{y} / N\right)\right]$ $\propto \exp \left\{\beta\left[E \Sigma_{x} \sigma_{x}+\left(\Sigma_{x} \sigma_{x}\right)^{2} / 2 N\right]\right\}$.

All this suggests the following classifications of our damage models.

(1) If the coupling constant is small $(\alpha \ll 1)$, the damage model is close to the percolation model so that in the approach to the transition at elongation $l_{c}$, the correlation length behaves as $\xi \sim\left|l-l_{c}\right|^{-\nu}$, with $\nu=4 / 3$.

(2) For non-negligible coupling constants $\alpha$, in the shorter-range case $\gamma>D$, we recover the same three possible behaviors as described above for the local load sharing rule.

(3) In the longer-range case where $\gamma<D$, we again recover the same four types of scenarios depending on the ratio $\beta\left(l_{c}\right) / A_{c}$ (as discussed in the local load sharing case). The model behaves as percolation when $\alpha \ll 1$. Otherwise, the behavior is determined by the ratio $\beta\left(l_{c}\right) / A_{c}$. Note that $A_{c}$ is of order unity, but depends on the particular exponent $\gamma$ and on the system size. If $\beta\left(l_{c}\right) / A_{c}<1$, the system behaves continuously and no transition is observed. If $\beta\left(l_{c}\right) / A_{c}>1$, there is a discrete jump in both the average number of broken cells and the sustained load. The correlation length remains finite in both of these two cases. Only the limiting case of $\beta\left(l_{c}\right) / A_{c} \sim 1$ corresponds to a second-order phase transition. In any of these cases, for large enough systems, the CurieWeiss description holds according to Refs. [46-48]. Accordingly, if there is any divergence of correlation length due to the system coming close to $\beta\left(l_{c}\right) / A_{c} \sim 1$ at $E=0$, the associated exponents should be those of the Curie-Weiss meanfield critical point $(\nu=1 / 2)$, and not the Ising one.

These results can be compared to numerical-simulation results in the literature for related models. In fiber-bundle models with power-law interactions [15], a transition has been found as a function of the interaction exponent $\gamma$ that is consistent with the above analysis, predicting mean-field behavior for the long-range case $\gamma<D$, and Ising-like behavior in the short-range case $\gamma>D$. Typical configurations prior to breakdown for this type of system are displayed in Fig. 5 of Ref. [15], and look very similar to percolation configurations close to the transition in the case $\gamma=0$, displaying smaller and smaller cluster sizes (characteristic of the autocorrelation length) and compact configurations as $\gamma$ increases above $D$. This is coherent with a mean-field behavior close to percolation transition in the first case, as opposed to a first-order transition (analogous to Ising model crossing $E=0$ below $T_{c}$ ) when $\gamma<D$. This analogy is even more apparent in Fig. 7, bottom, of Ref. [16], where an extension of this model was considered, with time-delayed fiber breaking process in addition to power-law decaying interactions [16].

Burned fuse models, in which the interactions between burned fuses decay as $1 / r^{D}$, exhibit diverging autocorrelation lengths at breakdown, with $\xi \sim\left|l-l_{c}\right|^{-\nu}$, where $l$ is the imposed voltage, and $\nu$ is equal to the percolation exponent [44], $\nu=4 / 3$ in 2D [22,50] or 0.88 in 3D [51]. Roux et al. [50] showed that in the limit of "infinitely large" disorder, such models are isomorphic to percolation models. When the disorder is less pronounced, stress concentration leads to regimes of localizing damage. At low disorders, stress concentration entirely dominates, and fuses burn in clusters of size 
scaling as the lattice step, leading to a so-called "total localization regime." At intermediate disorders, fuses burn essentially in regions that scale as the lateral size of the system (size in the direction perpendicular to the main current flow), a priori much larger than the lattice step, which constitutes the "soft localization regime" $[22,52]$. It has been shown $[22,51]$ that such a process is still in the percolation universality class, in the sense that there is still a growing autocorrelation length with an exponent identical to the percolation one. Based on the fact that these soft localizing regimes are in the percolation universality class, coupled with the largescale profile of damage in the system, Hansen et al. [22] related the roughness of the final fracture spanning through the system to the autocorrelation length exponent, based on arguments of percolation in a gradient, and this properly predicts the Hurst exponent of the final damage fronts, in both 2D and 3D. The morphology of the connected "fracture" at breakdown is oriented, and different from percolating clusters in the percolation model. This is related to the anisotropic character of interactions in the burned fuse model; i.e., the current perturbation from a burned fuse varies as a dipolar field decaying as $1 / r^{D}$, but also having an orientational aspect not included in the models under study in this paper. This anisotropic aspect is absent from the models discussed here, but the fact that the autocorrelation length exponent is similar to the ones of percolation is coherent with the fact that long-range systems are in the percolation universality class. Burned fuse systems are at the overlap between shortand long-range interactions, in the sense that they correspond to $\gamma=D$.

Last, fiber bundles connected to elastic plates, where $\gamma$ $=1$ and $D=2$, have been numerically studied in Ref. [28] and an autocorrelation length exponent of $\nu \simeq 1.54$ numerically determined. The discrepancy between this result and the percolation or Curie-Weiss critical-point result might presumably result from finite-size effects making this model $(\gamma$ $=1$ ) still significantly different from the Curie-Weiss one $(\gamma=0)$, or from the fact that the stress perturbation in this numerical model might have been too large for the interactions to be considered weak (a requirement for the validity of the present analysis).

\section{CONCLUSIONS}

We have treated a class of damage models having weak isotropic interactions between cells that become damaged in the lattice. A quenched disorder is present in the rupture energies for each lattice cell, and the evolution of damage is ruled by the Griffith principle. Averaging over all possible realizations of the underlying quenched disorder, the probability distribution of each possible damage configuration was obtained as a function of the deformation externally applied to the system.

The exact calculation is analytically tractable in the case of a global load sharing model, and it has been shown to be isomorphic to a percolation model. This corresponds to the behavior of a system totally dominated by the underlying disorder, where the next cell to break is always the weakest one. Spatial interactions added to the system modify this picture, since the overload created by broken cells induces some degree of spatial ordering that competes with the weakest cell mechanism. By limiting ourselves to small overloads compared to the average load of the system, it was possible to obtain the probability of damage configurations as integrated over all realizations of the quenched disorder.

In this weak interaction limit, the resulting probability distributions were shown to be Boltzmannians in the number of broken cells and in the stored elastic energy. This type of distribution maximizes Shannon's entropy under constraints related to the energetic balance of the fracture process, and we have demonstrated the formal relationship between our quenched-disorder damage models and the standard distributions arising in equilibrium statistical mechanics. This then allows the standard toolbox of statistical mechanics to be applied to our damage models.

Our systems map onto three types of possible behaviors: (1) percolation models in the case of interactions so weak they may be neglected; (2) Ising models for non-negligible short-range interactions; and (3) Curie-Weiss mean-field theory for non-negligible long-range interactions. The temperature and external field in the partition function of our models are analytical functions that depend on the particular sharing rule, on the type of quenched disorder considered, and on the average elongation (or deformation) externally loaded onto the system. The path followed in the Ising control parameter space when the load is increased from 0 depends on the qd distribution and the load sharing rule. When the formal external field changes sign, a phase transition is possible. This can correspond to a first-order phase transition, a percolationlike transition, or an Ising critical-point transition, depending on the value of the formal temperature during the transition.

The systems studied here are limited to isotropic load perturbations. We have earlier studied oriented crack models in [39-41], which correspond to anisotropic load perturbations that depend on the orientation of the crack opened in the lattice. Those earlier studies were based on an entropymaximum assumption. The hypotheses of the present work extend directly to oriented systems, and so the present paper justifies the entropy-maximum assumption postulated in our earlier work. The precise value of the temperature, and the physical interpretation of the functional forms given in [39-41], should be modified according to the results of this paper. Such modifications will, however, result in identical functional forms relating the configuration space and the PDF over configurations, and thus the present work confirms the existence of a phase transition in such an oriented crack model, with an associated divergence exponent of the autocorrelation length $\nu=2$.

Experimentally, the emergence of correlated damage at large scale has an important influence on the macroscopic rupture of a heterogeneous object. For example, the roughness of natural fracture surfaces, whose universality has long been observed experimentally [53], has recently been related to the behavior of the divergence of the damage correlation length at the approach to breakdown [22]. Damage correlated at large scale should also leave a signature on elastic wave scattering through the damaged material. The knowledge of the behavior of a damage autocorrelation function, as frac- 
turing progresses, is thus experimentally relevant in monitoring the approach to macroscopic failure or shear banding through the use of elastic-scattering experiments.

\section{APPENDIX: RECOVERABLE ENERGY AS A FUNCTION OF THE DAMAGE STATE}

The argument here will be specific to a fiber bundle model. However, as noted in the text, other weak damage models will also be controlled by the same type of storedenergy function obtained here.

Define a fiber bundle as (initially) $N$ fibers stretched between a free rigid plate and an elastic half space. The rigid plate has a controlled displacement $l$ applied to it that stretches the fibers and the elastic half space. As a fiber breaks at fixed $l$, the force it held will be transmitted to the other fibers through the elastic half space.

A fiber at point $\mathbf{x}$ is stretched a distance $\ell_{\mathbf{x}}$. Where that fiber is attached to the elastic half space, the surface of the half space is displaced by an amount $u_{\mathbf{x}}$. Thus, at those places $\mathbf{x}$ where fibers exist, we have

$$
l=\ell_{\mathbf{x}}+u_{\mathbf{x}} .
$$

The fiber at point $\mathbf{x}$ exerts a force on the elastic half space that is

$$
\frac{f_{\mathbf{x}}}{A_{F}}=Y_{F}\left(1-\varphi_{\mathbf{x}}\right) \frac{\ell_{\mathbf{x}}}{L_{F}},
$$

where $A_{f}$ is the cross-sectional area of the fiber (assumed to be independent of the extension), $L_{F}$ is the inital length of each fiber, and $Y_{F}$ is the Young's modulus of each fiber. The local order parameter $\varphi_{\mathbf{x}}$ is 0 if the fiber is intact and 1 if broken.

The Green function for point forces acting on the surface of an elastic half space [54] yields

$$
\begin{aligned}
u_{\mathbf{x}}=u_{3}(\mathbf{x}) & =\frac{1-\sigma_{s}^{2}}{\pi Y_{s}} \sum_{\mathbf{y} \neq \mathbf{x}} \frac{f_{\mathbf{y}}}{|\mathbf{y}-\mathbf{x}|} \\
& =\frac{1-\sigma_{s}^{2}}{\pi} \frac{Y_{F}}{Y_{s}} \frac{A_{F}}{L_{F}} \sum_{\mathbf{y} \neq \mathbf{x}} \frac{\left(1-\varphi_{\mathbf{y}}\right)}{|\mathbf{y}-\mathbf{x}|} \ell_{\mathbf{y}},
\end{aligned}
$$

where $Y_{s}$ is the Young's modulus and $\sigma_{s}$ the Poisson's ratio of the elastic solid. In general, the displacement at a point $\mathbf{x}=\left(x_{1}, x_{2}, x_{3}\right)$ within the elastic solid (where $x_{3}=0$ defines the surface) due to a point force acting at a point $\mathbf{y}=\left(y_{1}, y_{2}, 0\right)$ on the surface [i.e., $\mathbf{f}(\mathbf{x})=f_{\mathbf{y}} \delta(\mathbf{x}-\mathbf{y}) \hat{\mathbf{3}}$ ] is given by

$$
\begin{gathered}
u_{1}=\frac{1+\sigma_{s}}{2 \pi Y_{s}}\left[\frac{\left(x_{1}-y_{1}\right) x_{3}}{r^{3}}-\frac{\left(1-2 \sigma_{s}\right)\left(x_{1}-y_{1}\right)}{r\left(r+x_{3}\right)}\right] f_{\mathbf{y}}, \\
u_{2}=\frac{1+\sigma_{s}}{2 \pi Y_{s}}\left[\frac{\left(x_{2}-y_{2}\right) x_{3}}{r^{3}}-\frac{\left(1-2 \sigma_{s}\right)\left(x_{2}-y_{2}\right)}{r\left(r+x_{3}\right)}\right] f_{\mathbf{y}}, \\
u_{3}=\frac{1+\sigma_{s}}{2 \pi Y_{s}}\left[\frac{x_{3}^{2}}{r^{3}}+\frac{2\left(1-\sigma_{s}\right)}{r}\right] f_{\mathbf{y}},
\end{gathered}
$$

where

$$
r=\left[\left(x_{1}-y_{1}\right)^{2}+\left(x_{2}-y_{2}\right)^{2}+x_{3}^{2}\right]^{1 / 2} .
$$

Putting $x_{3}=0$ in the expression for $u_{3}(\mathbf{x} ; \mathbf{y})$ and then summing over all $\mathbf{y}$ yields the expression for the displacement $u_{\mathbf{x}}$ of the surface.

We now define the dimensionless number

$$
\varepsilon=\frac{\left(1-\sigma_{s}^{2}\right)}{\pi} \frac{Y_{F}}{Y_{s}} \frac{A_{F}}{L_{F} L_{P}},
$$

where a length $L_{P}$ has been defined as

$$
\frac{1}{L_{P}}=\sum_{\mathbf{y} \neq \mathbf{x}} \frac{1}{|\mathbf{y}-\mathbf{x}|}
$$

i.e., this sum is independent of which point $\mathbf{x}$ is considered. Assuming either that the elastic half space is stiffer than the fibers, or that each fiber has a length much greater than its width, or that fibers are spaced far enough apart that $L_{p}$ is large, allows $\varepsilon$ to be considered a small number. Since the fiber bundle is assumed to be made of a finite number $N$ of fibers, there is no divergence to $L_{P}$.

Using these definitions along with $u_{\mathbf{x}}=l-\ell_{\mathbf{x}}$ and iterating Eq. (A4) once to get the leading order in $\varepsilon$ contribution gives

$$
\frac{\ell_{\mathbf{x}}}{l}=1+\varepsilon\left(-1+\sum_{\mathbf{y} \neq \mathbf{x}} \frac{L_{P}}{|\mathbf{y}-\mathbf{x}|} \varphi_{\mathbf{y}}\right)+O\left(\varepsilon^{2}\right) .
$$

The elastic strain energy reversibly stored in each surviving fiber is then

$$
\begin{aligned}
E_{\mathbf{x}}=\frac{1}{2} f_{\mathbf{x}} \ell_{\mathbf{x}}= & \frac{1}{2} \frac{A_{F} Y_{F}}{L_{F}} l^{2}\left(1-\varphi_{\mathbf{x}}\right)\left(\frac{\ell_{\mathbf{x}}}{l}\right)^{2} \\
= & \frac{1}{2} \frac{A_{F} Y_{F}}{L_{F}} l^{2}\left\{1-\varphi_{\mathbf{x}}+2 \varepsilon\left[-1+\varphi_{\mathbf{x}}\right.\right. \\
& \left.\left.+\sum_{\mathbf{y} \neq \mathbf{x}} \frac{L_{P}}{|\mathbf{y}-\mathbf{x}|} \varphi_{\mathbf{y}}\left(1-\varphi_{\mathbf{x}}\right)\right]\right\}, \quad
\end{aligned}
$$

where terms of $O\left(\varepsilon^{2}\right)$ have been dropped. Thus, upon summing over all the fibers we obtain the total energy $E_{F}$ stored in the fibers as a function of the damage state

$$
\begin{aligned}
E_{F}=\sum_{\mathbf{x}} E_{\mathbf{x}}= & \frac{1}{2} \frac{A_{F} Y_{F}}{L_{F}} l^{2}\left[(1-2 \varepsilon) N-(1-4 \varepsilon) \sum_{\mathbf{x}} \varphi_{\mathbf{x}}\right. \\
& \left.-\varepsilon \sum_{\mathbf{x}, \mathbf{y} \neq \mathbf{x}} J_{\mathbf{x y}} \varphi_{\mathbf{x}} \varphi_{\mathbf{y}}\right],
\end{aligned}
$$

where the coupling constant is defined as

$$
J_{\mathbf{x y}}=\frac{2 L_{P}}{|\mathbf{y}-\mathbf{x}|} .
$$

This form of the fiber energy is consistent with what was defined in the text.

We now demonstrate that the energy recoverably stored in the elastic solid makes no important modification to $E_{F}$. The strain energy in the solid is given by 


$$
E_{s}=\frac{Y_{s}}{2\left(1+\sigma_{s}\right)} \int_{x_{3}>0} d^{3} \mathbf{x} \sum_{\mathbf{y} \neq \mathbf{x}}\left(e_{i k} e_{i k}+\frac{\sigma_{s}}{1-2 \sigma_{s}} e_{l l}^{2}\right),
$$

where summation over the indices is assumed and where the strain tensor is defined as

$$
e_{i k}(\mathbf{x} ; \mathbf{y})=\frac{1}{2}\left(\frac{\partial u_{i}(\mathbf{x} ; \mathbf{y})}{\partial x_{k}}+\frac{\partial u_{k}(\mathbf{x} ; \mathbf{y})}{\partial x_{i}}\right) .
$$

The displacements are given by Eqs. (A5)-(A7).

From these equations, the strain at points $\mathbf{x}$ inside the elastic solid takes the leading order in $\varepsilon$ form

$$
e_{i k}=\varepsilon l L_{P}\left[c_{i k}+\varepsilon \sum_{\mathbf{y} \neq \mathbf{x}} \frac{f_{i k}(\mathbf{x}-\mathbf{y})}{|\mathbf{x}-\mathbf{y}|^{2}} \varphi_{\mathbf{y}}\right],
$$

where the constant tensor $c_{i k}$ has units of inverse-length squared and the average strain tensor throughout the elastic solid is $\varepsilon l L_{P} c_{i k}$. The perturbation term due to the broken fiber volume integrates to zero. The tensor $f_{i k}$ has no dependence on the norm $|\mathbf{x}-\mathbf{y}|$; however, this fact is immaterial since $f_{i k}$ plays no important role.

Upon forming the required products for the integrand in Eq. (A16), and using the fact that terms linear in the brokenfiber perturbations integrate to zero, one obtains that the energy stored in the elastic solid is

$$
E_{s}=\frac{1}{2} \frac{A_{F} Y_{F}}{L_{F}} l^{2} \varepsilon V L_{p}\left(c_{i k} c_{i k}+\frac{\sigma_{s}}{1-2 \sigma_{s}} c_{l l}^{2}\right)\left[1+O\left(\varepsilon^{2}\right)\right]
$$

where $V$ is the volume integrated over (assumed finite). In other words, any energy stored in the elastic solid that is due to the interaction between fibers is $\varepsilon^{2}$ smaller than the leading order contribution which itself can be considered small. The leading order contribution depends only on the average number of broken fibers and thus does not alter the analytical form of Eq. (A14). Thus, the energy stored in the elastic solid plays no essential role in the damage model.
[1] A. A. Griffith, Philos. Trans. R. Soc. London, Ser. A 221, 163 (1920).

[2] Statistical Models for the Fracture of Disordered Media, edited by H. J. Herrmann and S. Roux (North-Holland, Amsterdam, 1990).

[3] H. E. Daniels, Proc. R. Soc. London, Ser. A 183, 404 (1945).

[4] H. E. Daniels, Adv. Appl. Probab. 21, 315 (1989).

[5] B. D. Coleman, J. Appl. Phys. 29, 968 (1958).

[6] F. T. Peirce, J. Text. Inst. 17, 355 (1926).

[7] D. Sornette, J. Phys. A 22, L243 (1989).

[8] D. Sornette, J. Phys. I 2, 2089 (1992).

[9] J. V. Andersen, D. Sornette, and K. T. Leung, Phys. Rev. Lett. 78, 2140 (1997).

[10] P. C. Hemmer and A. Hansen, J. Appl. Mech. 59, 909 (1992).

[11] S. R. Pride and R. Toussaint, Physica A 312, 159 (2002).

[12] A. Hansen and P. C. Hemmer, Phys. Lett. A 184, 394 (1994).

[13] M. Kloster, A. Hansen, and P. C. Hemmer, Phys. Rev. E 56, 2615 (1997).

[14] S. D. Zhang and E-jiang Ding, Phys. Rev. B 53, 646 (1996).

[15] R. C. Hidalgo, Y. Moreno, F. Kun, and H. J. Herrmann, Phys. Rev. E 65, 046148 (2002).

[16] O. E. Yewande, Y. Moreno, F. Kun, R. C. Hidalgo, and H. J. Herrmann, Phys. Rev. E 68, 026116 (2003).

[17] J. O. H. Bakke, J. Bjelland, T. Ramstad, T. Stranden, A. Hansen, and J. Schmittbuhl, Phys. Scr., T 106, 65 (2003).

[18] G. G. Batrouni and A. Hansen, Phys. Rev. Lett. 80, 325 (1998)

[19] L. de Arcangelis and H. J. Herrmann, Phys. Rev. B 39, 2678 (1989).

[20] L. de Arcangelis, A. Hansen, H. J. Herrmann, and S. Roux, Phys. Rev. B 40, 877 (1989).

[21] P. M. Duxbury, P. L. Leath, and P. D. Beale, Phys. Rev. B 36, 367 (1987).

[22] A. Hansen and J. Schmittbuhl, Phys. Rev. Lett. 90, 045504 (2003)
[23] A. Hansen, E. L. Hinrichsen, and S. Roux, Phys. Rev. B 43, 665 (1991).

[24] S. Zapperi, H. J. Herrmann, and S. Roux, Eur. Phys. J. B 17, 131 (2000).

[25] S. Zapperi, H. J. Herrmann, and S. Roux, Fractals 11, 327 (2003)

[26] G. G. Batrouni, A. Hansen, and J. Schmittbuhl, Phys. Rev. E 65, 036126 (2002).

[27] H. J. Herrmann, A. Hansen, and S. Roux, Phys. Rev. B 39, 637 (1989).

[28] J. Schmittbuhl, A. Hansen, and G. G. Batrouni, Phys. Rev. Lett. 90, 045505 (2003).

[29] Y. Moreno, J. B. Gomez, and A. F. Pacheco, Physica A 296, 9 (2001).

[30] Y. Moreno, J. B. Gómez, and A. F. Pacheco, Phys. Rev. Lett. 85, 2865 (2000).

[31] S. Zapperi, P. Ray, H. E. Stanley, and A. Vespignani, Phys. Rev. Lett. 78, 1408 (1997).

[32] S. Zapperi, P. Ray, H. E. Stanley, and A. Vespignani, Phys. Rev. E 59, 5049 (1999).

[33] S. Zapperi, A. Vespignani, and H. E. Stanley, Nature (London) 388, 658 (1997).

[34] F. Kun, S. Zapperi, and H. J. Herrmann, Eur. Phys. J. B 17, 269 (2000).

[35] D. Sornette and J. V. Andersen, Eur. Phys. J. B 1, 353 (1998).

[36] G. Caldarelli, F. D. Di Tolla, and A. Petri, Phys. Rev. Lett. 77, 2503 (1996).

[37] V. I. Räisänen, M. J. Alava, and R. M. Nieminen, Phys. Rev. B 58, 14288 (1998).

[38] J. B. Rundle and W. Klein, Phys. Rev. Lett. 63, 171 (1989).

[39] R. Toussaint and S. R. Pride, Phys. Rev. E 66, 036135 (2002).

[40] R. Toussaint and S. R. Pride, Phys. Rev. E 66, 036136 (2002).

[41] R. Toussaint and S. R. Pride, Phys. Rev. E 66, 036137 (2002).

[42] C. E. Shannon, Bell Syst. Tech. J. 27, 373 (1948).

[43] M. Barthelemy, R. da Silveira, and H. Orland, Europhys. Lett. 
57, 831 (2002).

[44] D. Stauffer and A. Aharony, Introduction to Percolation Theory, 2nd ed. (Taylor and Francis, London, 1994).

[45] K. Huang, Statistical Mechanics, 2nd ed. (Wiley, New York, 1987).

[46] S. A. Cannas, P. M. Gleiser, and F. A. Tamarit, available at http://tero.fis.uncor.edu/ ${ }^{\sim}$ cannas/papers/bookchapter3.pdf

[47] S. A. Cannas, A. C. N. de Magalhaes, and F. A. Tamarit, Phys. Rev. B 61, 11521 (2000).

[48] S. A. Cannas and F. A. Tamarit, Phys. Rev. B 54, R12661 (1996).

[49] Even if not all nonlocal models belong to the Curie-Weiss universality class: for example, the 1D Kac model, with inter- actions as $-L^{-1} \exp (-x / L)$, where the limit $L \rightarrow \infty$ is taken after the thermodynamic limit, obeys the van der Waals equation of state: C. J. Thompson, Classical Equilibrium Statistical Mechanics (Clarendon Press, Oxford, 1988), p. 95.

[50] S. Roux, A. Hansen, H. Herrmann, and E. Guyon, J. Stat. Phys. 52, 237 (1988).

[51] T. Ramstad, J. Ø. H. Bakke, J. Bjelland, T. Stranden, and A. Hansen, e-print cond-mat/0311606.

[52] R. Toussaint and A. Hansen, e-print cond-mat/0403217.

[53] E. Bouchaud, J. Phys.: Condens. Matter 9, 4319 (1997).

[54] L. D. Landau and E. M. Lifshitz, Theory of Elasticity, 3rd ed. (Pergamon Press, New York, 1986). 